\title{
A SHORT PROOF OF AN EXISTENCE THEOREM IN CONSTRUCTIVE MEASURE THEORY
}

\author{
Y. K. $\mathrm{CHAN}^{1}$
}

ABSTRACT. The most important example of an integration space in the constructive measure theory of Bishop and Cheng is the couple $(X, \mu)$, where $X$ is a locally compact metric space and $\mu$ is a nonnegative linear function on the space of continuous functions of compact support on $X$. Bishop and Cheng's proof that $(X, \mu)$ is indeed an integration space is rather involved. In this paper a much simpler proof is given.

Let $(X, d)$ be a locally compact metric space and let $\mu$ be a nonnegative linear function on the space $C$ of continuous functions on $X$ with compact supports. To rule out trivial cases assume that $\mu(f)>0$ for some $f$ in $C$. Bishop and Cheng [1] gave a constructive proof for the following

Theorem. If $\left(f_{m}\right)_{m=0}^{\infty}$ is a sequence of nonnegative functions in $C$ with $\sum_{m=1}^{\infty} \mu\left(f_{m}\right)$ convergent and less than $\mu\left(f_{0}\right)$, then there exists an $x$ in $X$ with $\sum_{m=1}^{\infty} f_{m}(x)$ convergent and less than $f_{0}(x)$.

This theorem is the hardest step in showing that $(X, \mu)$ is an integration space as defined in [1]. It has as consequences many existence theorems. The proof given in [1] is rather involved. We now give a much simpler proof.

Let $K$ be a compact set outside which $f_{0}$ vanishes. Construct a finite $1 / 2$-net $\left\{y_{1}, \cdots, y_{N}\right\}$ of $K$, and functions $j_{n}(1 \leq n \leq N)$ in $C$ with values in $[0,1]$ and such that $j_{n}(x)=1$ or 0 according as $d\left(x, y_{n}\right)<1 / 2$ or $>1$. Define $g_{1}=j_{1}$ and $g_{n}=g_{n-1} \vee j_{n}-g_{n-1}(1<n \leq N)$. Clearly $\Sigma_{1}^{N} g_{n} \leq 1$, and equality holds on $K$. Therefore, the hypothesis, $\Sigma_{m=1}^{\infty} \mu\left(f_{m}\right)<\mu\left(f_{0}\right)$, of the Theorem implies

$$
\sum_{n=1}^{N} \sum_{m=1}^{\infty} \mu\left(f_{m} g_{n}\right)<\sum_{n=1}^{N} \mu\left(f_{0} g_{n}\right)
$$

Hence there exists some $n(1 \leq n \leq N)$ such that $\sum_{m=1}^{\infty} \mu\left(f_{m} g_{n}\right)<\mu\left(f_{0} g_{n}\right)$.

Received by the editors March 5, 1974 .

AMS (MOS) subject classifications (1970). Primary 02E99, $28 \mathrm{~A} 30$.

Key words and phrases. Constructive analysis, Daniell integrals.

${ }^{1}$ Research partially supported by NSF Grant GP-29233. 
The function $h_{1} \equiv g_{n}$ vanishes outside a compact set of diameter at most 1 . Applying a similar argument to the last inequality, we construct inductively a sequence $\left(h_{k}\right)$ of nonnegative functions in $C$ where $h_{k}$ is supported by a compact set of diameter at most $k^{-1}$, and such that

$$
\sum_{m=1}^{\infty} \mu\left(f_{m} h_{1} \cdots h_{k}\right)<\mu\left(f_{0} h_{1} \cdots h_{k}\right) \quad(k \geq 1) .
$$

We next observe that if $f \in C$ is such that $\mu(f)>0$ then there exists $x \in X$ with $f(x)>0$. This observation together with $\sum_{m=1}^{k} \mu\left(f_{m} h_{1} \cdots h_{k}\right)<$ $\mu\left(f_{0} h_{1} \cdots h_{k}\right)$ immediately yields for each $k$ some $x_{k} \in X$ with

$$
0 \leq \sum_{m=1}^{k} f_{m} h_{1} \cdots h_{k}\left(x_{k}\right)<f_{0} h_{1} \cdots h_{k}\left(x_{k}\right) \text {. }
$$

Because $h_{k}$ has support of diameter at most $k^{-1}$, the above inequality implies $d\left(x_{k}, x_{j}\right) \leq k^{-1}$ if $k \leq j$. Hence $x_{k} \rightarrow x$ for some $x \in X$. But $K$ is a compact set outside which $f_{0}$ vanishes. Thus the $x_{k}$ 's and, therefore, $x$ belong to $K$. Because the $h_{k}$ 's are nonnegative, the last displayed inequality implies $\sum_{m=1}^{j} f_{m}\left(x_{k}\right)<f_{0}\left(x_{k}\right)(j \leq k)$. Letting $k \rightarrow \infty$, we have $\Sigma_{m=1}^{j} f_{m}(x)$ $\leq f_{0}(x)(j=1,2, \ldots)$. The reasoning up to this point is standard. However, the last inequality is not sufficient to guarantee the constructive convergence of $\sum_{m=1}^{\infty} f_{m}(x)$. Hence the necessity of the following argument.

Let $g \geq 0$ be a function in $C$ which equals 1 on $K$. Let $a>0$ be so small that $\sum_{m=1}^{\infty} \mu\left(f_{m}\right)+a+a \mu(g)<\mu\left(f_{0}\right)$. Let $N_{1}<N_{2}<\cdots$ be integers such that $\Sigma_{N_{k}}^{\infty} \mu\left(f_{m}\right)<2^{-2 k} a$. Then the first part of the proof can be applied to the sequence

$$
\left(f_{0}, a g, f_{1}, 2 \sum_{N_{1}}^{N_{2}} f_{m}, f_{2}, 2^{2} \sum_{N_{2}}^{N_{3}}, \cdots\right)
$$

to yield $x \in K$ such that

$$
a g(x)+f_{1}(x)+\cdots+f_{j}(x)+2^{j} \sum_{N_{j}}^{N_{j+1}} f_{m}(x) \leq f_{0}(x) \quad(j=1,2, \cdots) .
$$

In particular

(a) $\Sigma_{N j}^{N+1} f_{m}(x) \leq 2^{-j} f_{0}(x)$ and so $\Sigma_{1}^{\infty} f_{m}(x)$ converges;

(b) $a g(x)+\Sigma_{1}^{\infty} f_{m}(x) \leq f_{0}(x)$;

(c) $a g(x)=a>0$ and so $\Sigma_{1}^{\infty} f_{m}(x)<f_{0}(x)$.

The Theorem is proved. 


\section{REFERENCE}

1. E. Bishop and H. Cheng, Constructive measure theory, Mem. Amer. Math. Soc. No. 116 (1972).

DEPARTMENT OF MATHEMATICS, UNIVERSITY OF WASHINGTON, SEATTLE, WASH • INGTON 98195

Current address: Department of Mathematical Sciences, New Mexico State University, Las Cruces, New Mexico 88003 